\title{
Effects of Guizhi-Longgu-Muli Tang Supplementation on Physiopsychological Responses in Mental Stimuli 補充桂枝龍骨特螗湯於心理壓力下對生理心理反應之影響
}

\author{
Hsin-I LO ${ }^{1}$ Cheng-Hsien LIN $^{2}$ Mei-Yen CHEN $^{3}$ \\ Wen-Tzu TANG ${ }^{4}$ Mei-Chich HSU $^{5}$ \\ ${ }^{I}$ Graduate Institute of Physical Education, \\ National Taiwan Sport University, TAIWAN \\ ${ }^{2}$ Department of Sports Training Science-Athletics, \\ National Taiwan Sport University, TAIWAN \\ ${ }^{3}$ Department of Recreation and Leisure Industry Management, \\ National Taiwan Sport University, TAIWAN \\ ${ }^{4}$ Graduate Institute of Coaching Science, \\ National Taiwan Sport University, TAIWAN \\ ${ }^{5}$ Graduate Institute of Sports Science, National Taiwan Sport University, TAIWAN
}

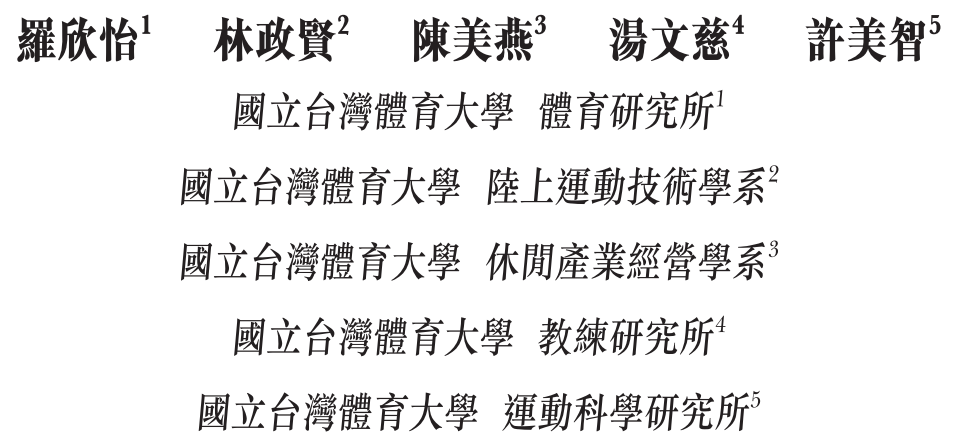

\begin{abstract}
The objective of the present study was to examine the effects of Guizhi-Longgu-Muli Tang (a traditional Chinese medicine) supplement on physiopsychological responses in mental stimuli in personal archery competition. Eleven archery athletes were randomly allocated into two groups: Guizhi-Longgu-Muli Tang (GLMT group) and Yi-Ren (placebo, PL group). All subjects completed 2 sessions following 3 days (2.0g/time, 3 times per day) of GLMT and placebo administered in a double-blind counterbalanced crossover manner with 14-day washout. Besides, the subjects also took 2.0g of GLMT or placebo $30 \mathrm{~min}$ before the personal archery competition. Our findings showed that no difference was seen between treatments in physical parameters of HR (heart rate), SBP (systolic blood pressure) before and after the competition. However, there was a significantly lower pre- and post-competition DBP (diastolic blood pressure) response in the GLMT trial than in the PL trial. In biochemical parameters, there was a significantly lower pre-competition GLU (glucose), ACTH (adrenocorticotropic hormone), and NE (norepinephrine) response in the GLMT trial than in the PL trial. Additionally, the trait anxiety was significantly positive correlated to state anxiety both in GLMT and PL groups. In conclusion, the findings of this study indicated that Guizhi-Longgu-Muli Tang supplementation could decrease the level of DBP, GLU, ACTH and NE. It might reduce physiopsychological responses in mental stimuli and probably improve exercise performance of the archery athletes.
\end{abstract}

Key words: Guizhi-Longgu-Muli Tang, mental stimuli, archery athletes, physiopsychological responses. 


\section{摘 要}

本研究主要探討服用桂枝龍骨生蠇湯後從事個人對抗賽對射箭選手生理心理反應之影響。以 11 位健康射箭選手為對象，採隨 機交叉平衡次序法, 分別給予每位受試者服用桂枝龍骨牡螈湯 (GLMT) 或安慰劑 (PL) 三天, 每天服用三次, 每次服用2.0g, 並於射箭對抗賽前 30 分鐘再服用 $2.0 \mathrm{~g}$, 之後在於射箭個人對抗賽前, 測量心跳率、血壓及測定促腎上腺皮質激素、皮質醇、腎上 腺素、正腎上腺素、睪固酮、生長激素及泌乳素等荷爾蒙的濃度。結果顯示, 服用桂枝龍骨牡蛕湯不影響競賽前後心跳率及收 縮壓; 但GLMT組舒張壓顯著低於PL組; GLMT組競賽前的血糖、促腎上腺皮質激素、正腎上腺素濃度顯著低於PL組; 此外, GLMT組與PL組的特質性焦虑與狀態性均呈正相關。綜合以上結果得知, 服用桂枝龍骨牡螰湯, 可降低競賽前的舒張壓、血糖、 促腎上腺皮質激素及正腎上腺素, 或許可降低競賽前的心理壓力, 進而促進運動表現。

關鍵字：桂枝龍骨牡蠇湯、心理壓力、射箭選手、生理心理反應

\section{Introduction}

The ancient Chinese remedy of Guizhi-LongguMuli Tang is a promising anti-anxiety drug, which is an ancient Chinese remedy for insomnia and anxiety. GuizhiLonggu-Muli Tang is a decoction of cassia twig, fossil fragments and oyster shells as the pharmaceutical remedy. It contains guizhi, longgu, muli, peony, gancao, ginger and dazao. Among these, longgu and muli show sedative effects. Longgu are fossilized fragments of animal bones and tortoise shells that, according to pharmacopoeia, function as a sedative. Longgu were recorded in the Shennong Bencao Jing (ca. 100 A.D.) and were especially valued for their treatment of spirit disorders. Dragon bone, usually combined with oyster shell (muli), figured prominently in the Shanghan Lun and companion text Jingui Yaolue (220 A.D.), particularly in the sedative formulas of Cinnamon and Dragon Bone Combination (Guizhi-Longgu-Muli Tang) and Bupleurum and Dragon Bone Combination (Chaihu-Longgu-Muli Tang). ChaihuLonggu-Muli Tang has similar indigenous medicines as Guizhi-Longgu-Muli Tang. It has been extensively accepted in a variety of clinical situations for the treatment of stress-related neuropsychiatric disorders (Yamada \& Kanba, 1997) and possesses an antidepressant-like activity in behavioral models (Zhu, Ma, Qu, \& Kang, 2006). ChaihuLonggu-Muli Tang, which is used in Japanese Kampo medical care for patients who have an oppressive feeling over the chest, insomnia and palpitations (Yamagiwa 1996) and may be identified as a promising inhibitor of smooth muscle cell proliferation and may be a promising candidate as a therapeutic agent in restenosis (Chung, Kim, Maruyama, \& Tani, 2003; Kim, Chung, Nose, Maruyama, \& Tani, 2002).
According to Spielberger (1966), the anxiety state is characterized by subjective, consciously perceived feelings of apprehension and tension, accompanied by or associated with activation or arousal of the autonomic nervous system. State anxiety can measure the temporary and situational anxiety state accompanied by autonomic excitement. The Trait scale assesses the tendency of an individual to respond to stressful circumstances under conditions of increased anxiety (Spielberger et al., 1970). Exposure to stressful stimuli is associated with the activation of the hypothalamic-pituitary-adrenal axis (HPA) and the release of catecholamines and glucocorticoid hormones(Udelsman \& Holbrook, 1994). Guizhi-LongguMuli Tang can affect the behavior and the endocrinal system of the chronic psychological stress rats by a certain level through the action on the different sectors of the hypothalamus-pituitary-adrenal gland axis (Zou et al., 2005).

Some plant medicines, show an antistress activity in behavioral tests in animals, which may be related to its normalization of the disturbed HPA axis (Li et al., 2001; Zhang, Yuan, Yang, Luo, \& Zhao, 2002). There is relatively little research that shows the benefit of GuizhiLonggu-Muli Tang in human beings. The objective of the present study was to examine the effects of GuizhiLonggu-Muli Tang supplementation on physiopsychological responses in mental stimuli of personal archery competition. Our study will clarify the interpretation of the physiopsychological mechanism factors. An expectation of this study is to develop an ideal coefficient relationship in advanced sports physiopsychological studies. 


\section{Methods}

\section{Subjects}

Eleven archery athletes, with a mean age $( \pm$ SE) of $20.6 \pm 1.5 \mathrm{yrs}$, weight $63.9 \pm 8.9 \mathrm{~kg}$, height of $168.7 \pm 7.3$ $\mathrm{cm}$, and trait anxiety questionnaire score $49.55 \pm 1.85$, participated in the experiment. The study conformed to the ethical guidelines of the National College of Physical Education and Sports (Taoyuan, Taiwan) and was approved by the Human Research Ethics Committee. All volunteers signed informed consent forms. In a pre-study interview, information on routine use of vitamins and other nutritional supplements was obtained from each participant. Volunteers found to be taking regular medication were excluded from the study. Two weeks prior to the tests, subjects were required to cease vitamin and supplement intake. Subjects were instructed to avoid exercise or strenuous physical activity for 3 days prior to the tests.

\section{Experimental design}

In this randomized counterbalance study, 11 subjects were allocated into two treatment groups, Guizhi-LongguMuli Tang (GLMT) and Yi-Ren (placebo, PL). The subjects were assigned to take either Guizhi-Longgu-Muli Tang or a placebo for three days. Guizhi-Longgu-Muli Tang and placebo were given at the dose of $2.0 \mathrm{~g} / \mathrm{time}$, 3 times per day. Besides, the subjects also took $2.0 \mathrm{~g}$ of Guizhi-Longgu-Muli Tang or placebo $30 \mathrm{~min}$ before the competition. A 14-day washout period followed before the subjects crossed over and received the alternate supplement for the next 3 days. They then completed a second competition.

Blood pressure, heart rate (HR), hormones were measured before and after taking Guizhi-Longgu-Muli Tang and $30 \mathrm{~min}$ before the archery stress condition. A competitive state anxiety questionnaire was also written before the competition on two sessions. The self-estimation questionnaire was written after the competition on three sessions.

\section{Stress Condition Design}

The stress condition was estimated in the one-byone archery competition. The subjects shot from $70 \mathrm{~m}$ at an $122 \mathrm{~cm}$ target face. This rhythm and timing applied to any of the matches. Archers shot six ends of three arrows (18 arrows), alternatively with a 40 sec- time limit per arrow.

\section{Psychological measures}

Ten minutes before each competition, trial subjects completed a State Anxiety (STAI) (Spielberger et al., 1970) questionnaire. The STAI questionnaire can be used to measure state or trait anxiety or both. The state anxiety inventory, measures state anxiety or how the subject feels "right now". The self-reporting inventory consists of 20 items to assess state anxiety, and another 20 items to assess trait anxiety. The response ranged from "not at all" (score 1) to "very much so" (score 4). High scores on their respective scales mean more trait or state anxiety and low scores mean less. Three parts of questions involve the self-estimation questionnaire.

\section{Blood collection and analyses}

Vein blood samples $(10 \mathrm{~mL})$ were collected from each subject before taking Guizhi-Longgu-Muli Tang and post-competition. Plasma from the samples was immediately centrifuged and the plasma frozen at $-20^{\circ} \mathrm{C}$ until assayed. Plasma glucose was determined at the appropriate wavelength, using a DT-60 analyzer (Johnson \& Johnson, Rochester, NY).

Hormones were detected by enzyme-linked immunosorbent assay (ELISA) using a commercially available kit (ActiveGLO ${ }^{\mathrm{TM}}$, Diagnostic Systems Laboratories, Inc., USA) according to the manufacturer's instructions.

\section{Statistical Analysis}

All data were expressed as mean \pm SEM (standard error of the mean) and analyzed by the paired t-test. Correlations between variables were examined by Pearson's correlation analysis. The results were considered significant when $\mathrm{p}<0.05$. Data were analyzed with SPSS 11.0.

\section{Results and Discussion}

Table 1 showed the physical parameters of the HR and SBP. There were no significant differences between two groups at the pre-competition and post-competition. DBP response of the GLMT group was significantly lower than the PL group during the pre-competition and post-competition. In biochemical parameters, the level of GLU, ACTH, and NE in the GLMT group were significantly lower than the PL group during precompetition. No significant differences were found in the measure of GH, TE and CORT during pre-competition and post-competition. 
Plasma ACTH secretion was taken as an indicator of HPA activation in response to acute stimulus. The activation of the HPA axis in response to experimental psychological stress is induced by central monoamines through CRF release(Clow, Patel, Najafi, Evans, \& Hucklebridge, 1997). Studies were conducted to understand how Guizhi-Longgu-Muli Tang affects the behavior and the endocrinal system of the chronic psychological stress rats in a certain level by through the action on the different sectors of the hypothalamus-pituitary-adrenal gland axis (Zou et al., 2005). Our study performed a sham archery competition; the results are justified due to the short time of the stress, showing that the stress did not significantly provoke HPA axis alteration.

Catecholamines are active amines containing catechol, which act as neurotransmitters and hormones. Such substances are biosynthesized from tyrosine. Catecholamines are controllers of the autonomous and central nervous system(Benedict, 1987). Studies conducted to understand the synthesis and metabolism of these monoamines during stress have been the main concern of many authors (Kvetnansky et al., 1993).

The adrenal glands present two portions, cortical and medullar, responsible for the glucocorticoids and catecholamines release, respectively. Circulating epinephrine stimulates norepinephrine release (Sanchez, Toledo-Pinto, Menezes, \& Pereira, 2003). The organic responses can be altered during stress situations, and at least partially, to be influenced by the hormones and/or neurotransmitters released in these stress situations. Moreover, other mechanisms involved in the transmission adrenergic can influence in the stress response (Graefe \& Henseling, 1983). The results of the present study indicate that NE concentration significantly increased in Guizhi-LongguMuli Tang group during pre-competition. It showed that $\mathrm{NE}$ released during acute psychical stress originates from the peripheral nerve endings in the sympathetic nervous system. It should be considered that the major stress response consists of a latency catecholamine response in the sympatho-adrenomedullary system and a short acting cortisol response in the hypothalamus-pituitary-adrenocortical axis (Sapolsky, Krey, \& McEwen, 1986).

The effects of Guizhi-Longgu-Muli Tang supplementation on psychosocial parameter and performance are listed in Table 2. There are no significant differences found in state anxiety, self-condition, confidence, self- examination and performance. Additionally, the trait anxiety was significantly positive correlated to state anxiety both in GLMT ( $\mathrm{r}=0.630 ; \mathrm{p}<.05)$ and $\mathrm{PL}$ groups $(\mathrm{r}=0.755$; $\mathrm{p}<.05)$. Thus, high trait anxiety would lead to an increase in state anxiety. Besides, the state anxiety was significantly positive correlated to EP in PL group but no significant differences were found in GLMT group. It seems that high state anxiety could stimulate EP secretion.

The present study did not reveal significant correlations between biochemistry parameter and the state anxiety variables but EP. Correlation analysis revealed that the importance of competition was positive correlated to the variables of state anxiety in two groups. Loupos et al. (2004) demonstrated that no significant changes were found in salivary cortisol concentration and cognitive anxiety during the championships. This finding was similar to our results. Other study found positive relationships between anxiety components (somatic and cognitive anxiety) and cortisol were noted in competition (Filaire, Sagnol, Ferrand, Maso, \& Lac, 2001). Two literatures (Caruso et al., 1990; Karteroliotis \& Gill, 1987), indicating that physiological and psychological measures of anxiety are not necessarily related.

In conclusion, the findings of this study indicated that Guizhi-Longgu-Muli Tang supplementation could possibly decrease the level of Glu, DBP, ACTH, and NE; it might reduce physiopsychological responses in mental stimuli and probably improve exercise performance of the archery athletes. Thus, with these results and the data from current study, we presume that the antistress activity of GLMT after a 3-day administration was probably consistent with its adaptogenic or antistress activity. Certainly, further studies are needed.

\section{Acknowledgements}

We wish to extend our deep appreciation to all participating subjects for their dedication to this study. The authors thank Dr. Chian-Hwa Chen for valuable comments on the manuscript. 
Table 1. Effects of Guizhi-Longgu-Muli Tang Supplementation on Physical Parameter and Hormone Responses.

\begin{tabular}{lllcc}
\hline \multirow{2}{*}{ Parameter } & \multicolumn{2}{c}{ Pre-competition } & \multicolumn{2}{c}{ Post-competition } \\
\cline { 2 - 5 } & \multicolumn{1}{c}{ GLMT } & \multicolumn{1}{c}{ PL } & GLMT & PL \\
\hline HR(beat/min) & $66.1 \pm 1.7$ & $65.7 \pm 1.6$ & $68.5 \pm 1.9$ & $65.7 \pm 1.6$ \\
SBP(mmHg) & $108.4 \pm 4.0$ & $111.6 \pm 2.7$ & $108.8 \pm 4.6$ & $111.6 \pm 2.7$ \\
DBP(mmHg) & $66.0 \pm 2.0^{\#}$ & $74.2 \pm 2.6$ & $67.4 \pm 2.3^{\#}$ & $74.2 \pm 2.6$ \\
GLU(mg/dL) & $86.3 \pm 3.9^{\#}$ & $93.9 \pm 1.7$ & $90.6 \pm 1.5$ & $93.9 \pm 1.7$ \\
ACTH(pg/mL) & $6.53 \pm 0.52^{\#}$ & $8.20 \pm 1.06$ & $8.12 \pm 1.19$ & $8.20 \pm 1.06$ \\
GH(ng/mL) & $2.30 \pm 1.09$ & $1.74 \pm 0.83$ & $1.10 \pm 0.49$ & $2.65 \pm 1.23$ \\
PRL(ng/mL) & $12.63 \pm 1.79$ & $10.01 \pm 1.23$ & $12.55 \pm 1.99$ & $10.12 \pm 1.19$ \\
TE(ng/mL) & $5.21 \pm 1.61$ & $3.91 \pm 0.70$ & $3.69 \pm 0.61$ & $3.44 \pm 0.58$ \\
CORT( $\mu$ g/dL) & $15.47 \pm 1.51$ & $15.40 \pm 1.80$ & $15.03 \pm 1.09$ & $14.73 \pm 1.67$ \\
EP(pg/mL) & $0.060 \pm 0.005$ & $0.066 \pm 0.006$ & $0.062 \pm 0.004$ & $0.068 \pm 0.006$ \\
NE(pg/mL) & $0.25 \pm 0.038^{*}$ & $0.30 \pm 0.045$ & $0.27 \pm 0.02$ & $0.23 \pm 0.03$ \\
\hline
\end{tabular}

- $\quad$ Mean \pm SEM ; $\mathrm{n}=11$

- GLMT: Guizhi-Longgu-Muli Tang group; PL: Placebo group; HR: heart rate; SBP: systolic blood pressure; DBP: diastolic blood pressure; GLU: glucose; ACTH : adrenocorticotropic Hormone; TE: testosterone; CORT: cortisol; EP: epinephrine; NE: norepinephrine; GH : growth hormone; PRL: prolactin

- $\quad$ Indicates significant difference to placebo group $(\mathrm{p}<0.05)$.

Table 2. Effects of Guizhi-Longgu-Muli Tang Supplementation on Psychosocial Parameter and Performance.

\begin{tabular}{ccccc}
\hline Group & State anxiety & Self-condition & Confidence & Self-examination \\
\hline GLMT & $42.3 \pm 4.1$ & $17.2 \pm 1.7$ & $12.7 \pm 1.3$ & $69.5 \pm 3.1$ \\
PL & $39.4 \pm 3.1$ & $18.5 \pm 2.2$ & $12.5 \pm 0.9$ & $68.1 \pm 4.0$ \\
\hline
\end{tabular}

- $\quad$ Mean \pm SEM ; $\mathrm{n}=11$

- GLMT: Guizhi-Longgu-Muli Tang group; PL: Placebo group 


\section{Reference}

Benedict, C. R. (1987). Simultaneous measurement of urinary and plasma norepinephrine, epinephrine, dopamine, dihydroxyphenylalanine, and dihydroxyphenylacetic acid by coupled-column highperformance liquid chromatography on $\mathrm{C} 8$ and $\mathrm{C} 18$ stationary phases. Journal of Chromatography, 385, 369-375.

Caruso, C., ZewaltOwski, D., Gill, D., \& McEiroy, M. (1990). Psychological and physiological changes in competitive state anxiety during noncompetition and competitive success and failure. Journal of Sport \& Exercise Psychology, 12, 6-20.

Chung, H. J., Kim, D. W., Maruyama, I., \& Tani, T. (2003). Effects of traditional Chinese formulations on rat carotid artery injured by balloon endothelial denudation. The American Journal of Chinese Medicine, 31(2), 201-212.

Clow, A., Patel, S., Najafi, M., Evans, P. D., \& Hucklebridge, F. (1997). The cortisol response to psychological challenge is preceded by a transient rise in endogenous inhibitor of monoamine oxidase. Life Science, 61(5), 567-575.

Filaire, E., Sagnol, M., Ferrand, C., Maso, F., \& Lac, G. (2001). Psychophysiological stress in judo athletes during competitions. The Journal of Sports Medicine and Physical Fitness, 41(2), 263-268.

Graefe, K. H., \& Henseling, M. (1983). Neuronal and extraneuronal uptake and metabolism of catecholamines. General Pharmacology, 14(1), 27-33.

Karteroliotis, C., \& Gill D. (1987). Temporal changes in psychological components of state anxiety. Journal of Sport \& Exercise Psychology, 9, 261-274.

Kim, D. W., Chung, H. J., Nose, K., Maruyama, I., \& Tani, T. (2002). Preventive effects of a traditional Chinese formulation, Chaihu-jia-Longgu-Muli-tang, on intimal thickening of carotid artery injured by balloon endothelial denudation in rats. The Journal of Pharmacy and Pharmacology, 54(4), 571-575.
Kvetnansky, R., Fukuhara, K., Pacak, K., Cizza, G., Goldstein, D.S., \& Kopin, I.J. (1993). Endogenous glucocorticoids restrain catecholamine synthesis and release at rest and during immobilization stress in rats. Endocrinology, 133(3), 1411-1419.

Li, Y. F., Yuan, L., Xu, Y. K., Yang, M., Zhao, Y. M., \& Luo, Z. P. (2001). Antistress effect of oligosaccharides extracted from Morinda officinalis in mice and rats. Acta Pharmacologica Sinica, 22(12), 1084-1088.

Loupos, D., Tsalis, G., Barkoukis, V., Semoglou, K., \& Mougios, V. (2004). Psychophysiological effects of competitive stress on swimming coaches. Journal of. Swimming Research, 16, 1-5.

Sanchez, A., Toledo-Pinto, E. A., Menezes, M. L., \& Pereira, O. C. (2003). Changes in norepinephrine and epinephrine concentrations in adrenal gland of the rats submitted to acute immobilization stress. Pharmacological Research, 48(6), 607-613.

Sapolsky, R. M., Krey, L. C., \& McEwen, B. S. (1986). The neuroendocrinology of stress and aging: the glucocorticoid cascade hypothesis. Endocrine Reviews, 7(3), 284-301.

Spielberger, C. D. (1966). Anxiety and behavior. New York: Academic press.

Spielberger, C. D., Gorsuch, R. L. \& Lushene, R. E. (1970). Manual for the state- trait anxiety inventory. Palo Alto, CA: Consulting Psychologists Press.

Udelsman, R., \& Holbrook, N. J. (1994). Endocrine and molecular responses to surgical stress. Current Problems in Surgery, 31(8), 653-720.

Yamada, K., \& Kanba, S. (1997). Kampo therapy and stress in the field of neuropsychiatry. Progress in Medicine. 17, 817-822.

Yamagiwa, M. (1996). The effects of Kampo Therapy on throat discomfort in patients with autonomic nervous dysfunction. Japanese Journal of Oriental Medicine, 46, 765-772. (In Japanese with English abstract) 
Zhang, Z. Q., Yuan, L., Yang, M., Luo, Z. P., \& Zhao, Y. M. (2002). The effect of Morinda officinalis How, a Chinese traditional medicinal plant, on the DRL 72-s schedule in rats and the forced swimming test in mice. Pharmacology, Biochemistry, and Behavior, 72(1-2), 39-43.

Zhu, W., Ma, S., Qu, R., \& Kang, D. (2006). Antidepressant-like effect of saponins extracted from Chaihu-jia-longgu-muli-tang and its possible mechanism. Life Science, 79(8), 749-756.

Zou, J., Liang, S. H., Zhao, C. H., Ni, L. Q., Cao, B. Y., Chen, H. J., \& Tong, Y. (2005). The effect of two kinds of traditional Chinese herbal compound on the behavior and hypothalamus-pituitary-adrenal gland axis of the chronic psychological stress rats. Journal of Jinan University, 26, 530-534.

\section{Correspondence:}

Dr. Mei-Chich Hsu

Graduate Institute of Sports Science, National

College of Physical Education \& Sports. Taoyuan County, Taiwan, R.O.C.

250, Wenhua 1st Rd, Gueishan, Taoyuan

County, Taiwan 333, R.O.C.

E-mail : meichich1224@mail2000.com.tw

Telephone: 886-3-3283201 ext. 2604 or 2421

Fax : 886-3-3311843 